\title{
BMJ Open Cohort profile: patient characteristics and quality-of-life measurements for newly-referred patients with atrial fibrillation-Keio interhospital Cardiovascular Studies-atrial fibrillation (KiCS-AF)
}

Nobuhiro Ikemura (D) , John A Spertus, ${ }^{2}$ Takehiro Kimura, ${ }^{1}$ Kenneth Mahaffey, ${ }^{3}$ Jonathan P Piccini, ${ }^{4}$ Taku Inohara, ${ }^{1,5}$ Ikuko Ueda, ${ }^{1}$ Kojiro Tanimoto, ${ }^{6}$ Masahiro Suzuki, ${ }^{7}$ Iwao Nakamura, ${ }^{8}$ Makoto Akaishi, ${ }^{9}$ Hideo Mitamura, ${ }^{10}$ Keiichi Fukuda, ${ }^{1}$ Seiji Takatsuki, ${ }^{1}$ Shun Kohsaka ${ }^{1}$

To cite: Ikemura N, Spertus JA, Kimura T, et al. Cohort profile: patient characteristics and quality-of-life measurements for newly-referred patients with atrial fibrillation-Keio interhospital Cardiovascular Studies-atrial fibrillation (KiCS-AF). BMJ Open 2019;9:e032746. doi:10.1136/ bmjopen-2019-032746

- Prepublication history and additional material for this paper are available online. To view these files, please visit the journal online (http://dx.doi. org/10.1136/bmjopen-2019032746).

Received 04 July 2019 Revised 09 0ctober 2019 Accepted 22 November 2019

A) Check for updates

C Author(s) (or their employer(s)) 2019. Re-use permitted under CC BY-NC. No commercial re-use. See rights and permissions. Published by BMJ.

For numbered affiliations see end of article.

Correspondence to

Dr Shun Kohsaka; sk@keio.jp

\section{ABSTRACT}

Purpose Besides the high rates of morbidity and mortality, atrial fibrillation (AF) is also associated with impairment of quality-of-life (QOL). However, reports covering non-selected AF population within Asian countries remain scarce. The objective of the Keio interhospital Cardiovascular Studies-atrial fibrillation (KiCS-AF) registry is to clarify the baseline and QOL profiles of the AF patients at the time of initial referral to identify areas for improvement and country-specific gaps.

Participants The KiCS-AF registry is a multicentre, prospective cohort study designed to specifically recruit AF patients newly referred to the 11 network hospitals within the Kanto area of Japan. The registry completed its enrolment in June 2018. All patients were requested to answer the Atrial Fibrillation Effect on Quality-of-Life (AFEQT) questionnaire both at baseline and 1 year, with planned clinical follow-up for 5 years. The registry also assessed individual treatment strategies including rate and rhythm control, stroke prophylaxis, and their impacts on patient-reported QOL.

Findings to date As of December 2016, 2464 AF patients were registered; their mean age was 67.1 years (SD, 11.7), majority (69.7\%; $n=1717)$ were men and $49.2 \%$ presented with paroxysmal AF. The mean $\mathrm{CHA}_{2} \mathrm{DS}_{2}-\mathrm{VASC}$ (cardiac failure or dysfunction, hypertension, age $\geq 75$ years, diabetes, stroke including vascular disease, age 65-74 years, and sex category [female]) score was 2.3 $(\mathrm{SD}, 1.6)$ and oral anticoagulant therapy was used for $88.6 \%$ of patients with $\mathrm{CHA}_{2} \mathrm{DS}_{2}-\mathrm{VASc}$ scores $\geq 2$. The median AFEQT-overall summary score was 79.1 (IQR, 66.6-89.1). Roughly $50 \%$ had significantly impaired QOL (ie, AFEQT <80) at baseline. Currently, 2307 eligible patients $(93.6 \%)$ have completed the 1-year follow-up, of which 2072 patients (89.8\%) answered the second AFEQT questionnaire.

Future plans The KiCS-AF allowed for extensive investigation of AF-related QOL in a non-selected population with long-term follow-up using a rigorously

\section{Strengths and limitations of this study}

The Keio interhospital Cardiovascular Studies-atrial fibrillation registry is a multicentre, registry-based prospective cohort study designed to specifically collect clinical information on newly-referred atrial fibrillation patients in Japan.

- All patients were requested to answer the Atrial Fibrillation Effect on Quality-of-Life questionnaire both at baseline and 1 year, with planned clinical follow-up for 5 years.

- To ensure consecutive case enrolment, a dedicated study coordinator and investigator performed onsite auditing to ensure proper registration of each eligible patient and all of the clinical endpoints were validated.

- Potential limitation includes observational design and generalisability of the study results (the study was conducted in University affiliated hospitals within Tokyo metropolitan area).

validated QOL assessment tool. Almost half of patients had impaired QOL at baseline. Further investigations aimed at providing care and improving patient-reported QOL are required.

\section{INTRODUCTION}

Atrial fibrillation (AF) is the most frequently encountered sustained arrhythmia and is associated with substantial morbidity and mortality and impaired quality-of-life (QOL) worldwide. ${ }^{1-4}$ Further, AF is known to constitute a significant public health problem, and estimates suggest that this condition accounts for $1 \%$ of the National Health Service budget in $\mathrm{UK}^{5}$ and $\$ 16-26$ billion of annual US 
healthcare expenditures. ${ }^{6}$ The incidence of AF is known to increase rapidly with advancing age and its burden is expected to continue to rise. ${ }^{7}$ An estimated 700000 people in Japan have AF, which is projected to increase to $>1$ million by $2050 .{ }^{89}$

Over the last decade, efficacy of new therapies for both stroke prevention (ie, non-vitamin $\mathrm{K}$ antagonist oral anticoagulants (NOACs)) and rhythm control has been demonstrated. ${ }^{10}$ Although these and other studies have advanced our knowledge of AF, many of the prior clinical studies and registries have focused on all-comer $\mathrm{AF}$ patients; hence their patient background varied significantly, and the implications for actual patient care remain limited. ${ }^{11}$ Moreover, data on the patient perception, including patient-reported outcomes (PROs) remain scarce; this is mainly due to relatively small sample sizes, restricted follow-up and the lack of validated AF-specific QOL assessments. Therefore, it is of utmost importance to clarify the profiles of patients, including the QOL assessment to identify country-specific gaps and areas for improvement, particularly in Japan, where social health insurance was implemented in $1961 .^{12}$

The Keio interhospital Cardiovascular Studies-atrial fibrillation (KiCS-AF) registry is a multicentre, prospective cohort study of patients with newly-diagnosed or referred AF. The registry collects information regarding patient-reported QOL and also assesses its association with individual treatment strategies, including rate and rhythm control, and stroke prophylaxis, within contemporary Japanese clinical practice. The present report describes the clinical characteristics of patients with newly-diagnosed or referred AF in Japan based on the preliminary data collected in this study, and the similarities and differences in characteristics of these patients compared with those in Western countries.

\section{COHORT DESCRIPTION \\ Registry objectives}

The objectives of the KiCS-AF registry are as follows: (1) describe non-selected AF patients in Japanese routine clinical practice, including patients' demographics, comorbidities and risk profiles using a contemporary scoring system; (2) explore the adoption and impact of emerging anticoagulant, rate-control and antiarrhythmic therapies (eg, catheter ablation for AF or use of antiarrhythmic drugs) on PROs and use of healthcare resources and (3) evaluate long-term clinical outcomes in patients with $\mathrm{AF}$ within contemporary Japanese practice.

\section{Participants}

Patients meeting inclusion criteria were registered by investigators (table 1). All patients more than 20 years old with electrocardiographically documented AF (eg, by ECG, Holter monitoring, implanted device and so on) were eligible, including patients with both valvular and non-valvular AF. Patients with atrial flutter alone were not eligible. The registry included patients with prevalent
Table 1 Inclusion and exclusion criteria

\begin{tabular}{ll}
\hline Inclusion criteria & Exclusion criteria \\
\hline Age $\geq 20$ years. & Atrial flutter only without AF. \\
Electrocardiographically & Current participation in a \\
confirmed AF. & randomised clinical study \\
Patients with prevalent & for AF. \\
or incident AF who were & Patients who are not \\
newly referred to the & considered suitable by the \\
network hospitals within & attending physicians. \\
the previous 6 months. & \\
Anticipated, regular & \\
annual follow-up & \\
outpatient visits. & \\
Signed informed & \\
consent. & \\
\hline
\end{tabular}

$\mathrm{AF}$, atrial fibrillation.

or incident $\mathrm{AF}$ who were newly referred to the network hospitals within the previous 6 months. A dedicated clinical research coordinator screened the patients from an administrative dataset provided monthly by each hospital, and query for study participation was sent to the physicians who set the appointments with patients under the new diagnostic coding of AF. When deemed eligible by the treating physicians, patients were requested to participate in the registry within 1-6 months from their initial visit. All participants provided written informed consent. Patients were enrolled both from inpatient and outpatient settings and patients with AF due to a reversible cause (eg, thyroid disease or postoperative AF) were included in this registry.

The KiCS-AF registry started enrolling patients in September 2012 and enrolment was completed by June 2018. A total of 11 institutions within the Tokyo metropolitan area in Japan (Saitama, Tochigi, Chiba, Kanagawa and Tokyo Prefecture) participated in the study; most are large tertiary care referral centres (figure 1; over $70 \%$ with $>500$ beds). The enrolment starting dates vary between participating hospitals since approval dates by the individual ethics committee differed. A total of 11 hospitals completed its enrolment in June 2018.

\section{Data collection and follow-up}

The primary data source was the medical records of registered patients. Dedicated clinical research coordinators were assigned and trained for the present registry; the coordinators have met biannually for updates and to discuss the queries from each institution. In collaboration with the primary site investigators (listed in the Collaborators section), coordinators were instructed to record and register data from consecutive hospital visits for patients with $\mathrm{AF}$ using an internet-based data collection system. Data quality assurance was achieved through automatic system validation and reporting of data completeness. PROs' questionnaires were aimed to be administered to all patients at the baseline visit and during follow-up visits or by mail. Yearly follow-up examinations were performed 


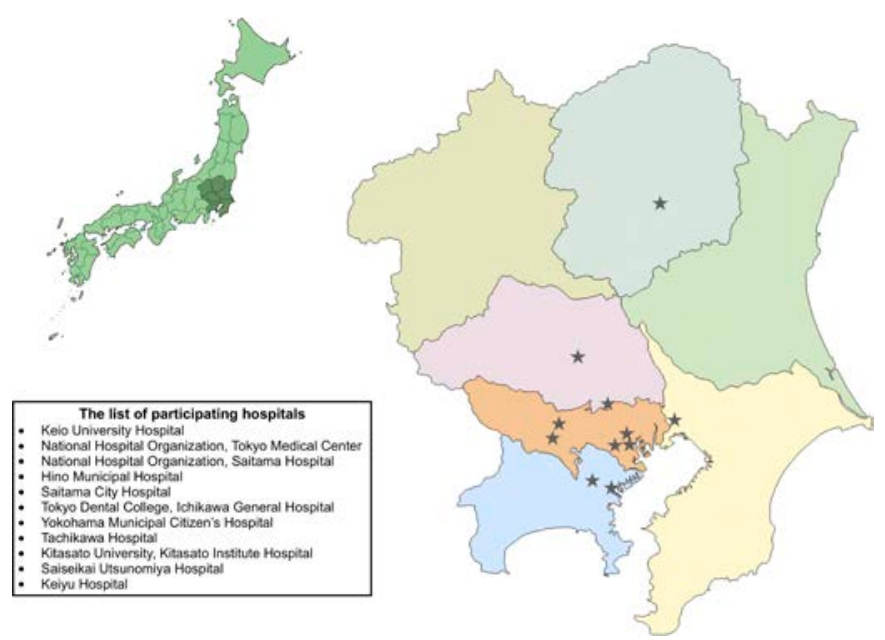

Figure 1 Geographic distribution of Keio interhospital Cardiovascular Studies-atrial fibrillation enrolling sites. A total of 11 hospitals completed its enrolment in June 2018 .

for all patients by mail, phone interviews and chart reviews. Trained study coordinators also transcribed the PROs' questionnaires and updated the status of major cardiovascular events and procedures and subsequent medication changes, and laboratory test values were collected.

Data collection was ensured at 1-year intervals for a minimum of 2 years after registration (table 2). Data collection windows were expanded to 6 months in either direction to maximise data collection during patients' follow-up visits. Baseline data include patient backgrounds, symptoms, prior and current drug use (including oral anticoagulants (OACs) and antiplatelet agents), ECGs, echocardiograms and blood sampling test results. Patient background factors included parameters for calculating risk scores for stroke, for example, congestive heart failure, hypertension, age $\geq 75$ years, diabetes mellitus and stroke for the CHADS $_{2}$ score $^{13}$; cardiac failure or dysfunction, hypertension, age $\geq 75$ years, diabetes, stroke including vascular disease, age 65-74 years and sex category (female) for the $\mathrm{CHA}_{2} \mathrm{DS}_{2}-$ VASc score. ${ }^{14}$ OACs included warfarin and direct OACs (ie, dabigatran, rivaroxaban, apixaban and edoxaban), and antiplatelet agents included aspirin, clopidogrel and prasugrel. Blood samples were analysed for complete blood count, liver function, kidney function, coagulation and serum brain natriuretic peptide level. In detail, data regarding anticoagulant therapies, dosage and discontinuations were collected as follow-up medication data. For patients prescribed warfarin, international normalised ratio values were collected. The definitions of each variable are available in the online supplementary material.

\section{Patient-reported outcomes}

All patients completed a detailed questionnaire regarding QOL and their perception of treatment at baseline and at 1-year follow-up by using the internationally validated Atrial Fibrillation Effect on Quality-of-Life (AFEQT; http://www.afeqt.org) questionnaire. The development and validation of AFEQT have been previously described. ${ }^{15}$ It is a 20 -item questionnaire that quantifies four domains of AF-related QOL including symptoms, daily activities, treatment concern and treatment satisfaction by using 7-point Likert response scales. An overall summary score can be calculated from the first three domains, which range from 0 to 100 (100, best possible health status (no impairment); 0, worst health status). A culturally and linguistically translated version of AFEQT for Japan was used. Furthermore, patients' subjective complaints, including palpitation, dyspnoea on exertion, difficulty of activities, dizziness, fatigue, chest pain and syncope, were evaluated by a physician at an initial medical interview in an outpatient clinic before access to patients' AFEQT questionnaires and immediately after enrolment.

\section{Outcomes}

The primary outcome event in KiCS-AF was stroke or systemic embolism. Stroke was defined as a new, sudden, loss of neurological function with residual symptoms lasting at least 24 hours after onset and is not due to a non-vascular cause (eg, seizure). Primary outcome events were confirmed by document submission and central review at the data coordinating centre. The primary

Table 2 Timeline of data collection in KiCS-AF

\begin{tabular}{|c|c|c|c|}
\hline & Baseline & 1 year & 2 years \\
\hline Vital signs, laboratory data, ECG and echo data & $\mathbf{X}$ & * & * \\
\hline Temporary and permanent discontinuation of antithrombotic therapies & & $\mathbf{X}$ & $\mathbf{X}$ \\
\hline Patient-reported outcomes (ie, AFEQT) & $\mathbf{X}$ & $\mathbf{X}$ & \\
\hline
\end{tabular}

The data on ECG only were obtained at each follow-up.

AF, atrial fibrillation; AFEQT, Atrial Fibrillation Effect on Quality-of-Life; KiCS-AF, the Keio interhospital Cardiovascular Studies-atrial fibrillation; PT-INR, prothrombin time-international normalised ratio. 
safety outcome of interest was major bleeding, as defined by the International Society of Thrombosis and Hemostasis criteria, ${ }^{16}$ which included bleeding events meeting at least one of the following criteria:

1. Fatal bleeding, and/or

2. Symptomatic bleeding in a critical area or organ (such as intracranial, intraspinal, intraocular, retroperitoneal, intra-articular or pericardial, or intramuscular with compartment syndrome), and/or

3. Bleeding causing a fall in haemoglobin level by $20 \mathrm{~g} / \mathrm{L}$ or more or leading to transfusion of two or more units of whole blood or red cells.

Secondary outcomes included all causes of death and hospitalisation. Death is classified into 1 of 3 categories: (1) cardiovascular death; (2) non-cardiovascular death and (3) undetermined cause of death. Cardiovascular death was subcategorised based on the cardiovascular aetiology of the death, which included acute myocardial infarction, sudden cardiac death, heart failure, stroke and other cardiovascular causes (eg, pulmonary embolism, aortic dissection, aortic aneurysm or peripheral arterial disease). Specific definitions of each outcome were described in online supplementary table 1 . All causes of hospitalisation were categorised as cardiovascular (eg, acute coronary syndrome, coronary revascularisation and acute heart failure) or non-cardiovascular hospitalisations. Use of AF-related procedures-including catheter ablation, pacemaker implantation, cardioversion, atrioventricular node ablation and left atrial appendage closure-were recorded for analysis.

\section{Sample size}

The goal of enrolling 3333 patients is to capture a large representative population of Japanese patients with AF. Based on recently completed clinical trials and observational studies, we anticipate an incident stroke rate of $2.4 \%-3.0 \%$ in our unselected KiCS-AF population. ${ }^{17} 18$ This would correspond to approximately 80 strokes by the end of the follow-up period. In addition, to compare patients' reported QOL scores in four analytic groups (two study groups (warfarin and non-warfarin) and two strata (incident and prevalent)), we will require 1500 patients in each group to have $80 \%$ power to detect a 2-point difference in the mean score of an item between two groups (assuming an SD of unity, a two-sided $\alpha=0.05$ )

\section{Statistical analyses}

Statistical plans varied according to specific analyses. Yet, there were certain considerations incorporated in all analyses of KiCS-AF. Importantly, several adjustment methodologies, including multivariable adjustment, or other methods have been considered when comparing patients. Continuous variables were summarised by medians with 25th and 75th percentiles, or by means with SD, and categorical variables were presented as numbers and percentages. Associations were reported using hazards ratios (eg, for COX regression models) or odds ratios (eg, for logistic regression models) with $95 \%$ confidence intervals. All reported $\mathrm{p}$ values were two-sided with a $\mathrm{p}$ value $<0.05$ being considered statistically significant.

\section{Patients involvement}

Patients and the public were not involved in the development of the research question, the design of the study, the recruitment and the conduct of the research.

\section{Information disclosure}

The KiCS-AF Steering Committee was responsible for overall study guidance, including the study protocol, data analysis and interpretation of results. Before the launch of the KiCS-AF registry, information regarding the objectives of the study and its social significance were provided for clinical trial registration with the University Hospital Medical Information Network (UMIN 000022229). This network is recognised by the International Committee of Medical Journal Editors as an 'acceptable registry' according to a statement issued in September 2004.

\section{FINDINGS TO DATE}

As of December 2016, a total of 2464 patients were enrolled from 11 institutions. Almost all patients agreed to participate. The number of patients who refused to participate was only available from a limited number of participating centres. The refusal rate was $3.1 \%$ (42/1322, online supplementary table 2). At that time, 2307 patients (93.6\%) had completed the 1-year follow-up, and 2072 patients $(89.8 \%$ ) had 1-year follow-up AFEQT data.

\section{Patients demographics within the KiCS-AF registry}

The characteristics of these patients are shown in table 3. The mean age was 67.1 years (SD, 11.7) and $69.7 \%$ $(n=1717)$ were men. Almost half $(49.2 \%)$ of the patients presented with paroxysmal $\mathrm{AF}$, while only $6 \%$ had firstdetected $\mathrm{AF}$. In addition, a quarter of patients had AF diagnosed during routine medical screening programme. The mean and median $\mathrm{CHA}_{2} \mathrm{DS}_{2}$-VASc scores in the KiCS-AF registry was 2.3 (SD, 1.6) and 2 (IQR, 1-3), respectively. OAC therapy was used for $88.6 \%$ of patients with stroke risk (ie, $\mathrm{CHA}_{2} \mathrm{DS}_{2}$-VASc score $\geq 2$ ), 1637 patients $(66.4 \%$ ) were prescribed NOACs, and 384 patients (15.6\%) were prescribed warfarin. Of note, almost half of the registered patients $(53.5 \%)$ opted for a rhythm control strategy.

The present report indicates that patients enrolled in the KiCS-AF were younger, more likely to be male, less likely to have cardiac comorbidities, and consequently had lower $\mathrm{CHA}_{2} \mathrm{DS}_{2}$-VASc scores, compared with previous reports from Western registries, such as Outcomes Registry for Better Informed Treatment of Atrial Fibrillation (ORBIT-AF) (table 4). ${ }^{19}$ Frequent diagnosis during the annual health screening programme (ie, typical Japanese workers are eligible to undergo annual health screenings that include a 12-lead ECG examination) may contribute to these differences. No data specifically addressed the risk of stroke and death in screen-detected $\mathrm{AF}$ in the general population. Future studies that ascertain the 
Table 3 Baseline characteristics

\begin{tabular}{|c|c|}
\hline Characteristics & $\begin{array}{l}\text { no. }(\%) \\
\text { nor }\end{array}$ \\
\hline Age, median (IQR), years & $68(60-75)$ \\
\hline Men & $1717(69.7)$ \\
\hline BMI, median, kg/m² (IQR) & $23.2(21.1-25.5)$ \\
\hline Heart rate, median, bpm (IQR) & $78(67-90)$ \\
\hline \multicolumn{2}{|l|}{ Blood pressure, median, $\mathrm{mm} \mathrm{Hg}$ (IQR) } \\
\hline Systolic & $128(117-139)$ \\
\hline Diastolic & $76(69-85)$ \\
\hline \multicolumn{2}{|l|}{ Medical history } \\
\hline Smoking & 407 (16.5) \\
\hline Hypertension & $1369(55.6)$ \\
\hline Diabetes mellitus & $402(16.3)$ \\
\hline Dyslipidaemia & $810(32.9)$ \\
\hline Heart failure & $385(15.6)$ \\
\hline Obstructive sleep apnoea & $73(3.0)$ \\
\hline Stroke or TIA & $211(8.6)$ \\
\hline Gastrointestinal bleeding & $34(1.3)$ \\
\hline CKD (eGFR <60 mL/min) & $1011(41.0)$ \\
\hline CKD on HD & $16(0.6)$ \\
\hline Peripheral artery disease & $82(3.3)$ \\
\hline Coronary artery disease & $196(8.0)$ \\
\hline \multicolumn{2}{|l|}{ Prior revascularisation } \\
\hline Prior PCl & $122(5.0)$ \\
\hline Prior CABG & $21(0.9)$ \\
\hline Prior valve surgery & $39(1.6)$ \\
\hline BNP, median, pg/mL (IQR) & $91.8(38.4-183.6)$ \\
\hline $\mathrm{CHADS}_{2}$ score, mean (SD) & $1.3(1.2)$ \\
\hline $\mathrm{CHADS}_{2}$ score, median (IQR) & $1(0-2)$ \\
\hline $\mathrm{CHA}_{2} \mathrm{DS}_{2}-\mathrm{VASc}$ score, mean (SD) & $2.3(1.6)$ \\
\hline $\mathrm{CHA}_{2} \mathrm{DS}_{2}-\mathrm{VASc}$ score, median (IQR) & $2(1-3)$ \\
\hline LVEF, median, \% (IQR) & $60(58.7-60)$ \\
\hline LA diameter, median, cm (IQR) & $4.1(3.6-4.6)$ \\
\hline \multicolumn{2}{|l|}{ Type of visit } \\
\hline Diagnosed at health screening & $642(26.1)$ \\
\hline Referral from emergency department & $224(9.1)$ \\
\hline \multicolumn{2}{|l|}{ Type of AF } \\
\hline First detected/new onset & $147(6.0)$ \\
\hline Paroxysmal & $1213(49.2)$ \\
\hline Persistent & $665(27.0)$ \\
\hline Permanent & $375(15.2)$ \\
\hline \multicolumn{2}{|l|}{ Current drug therapy } \\
\hline$\beta$-blockers & $1314(53.3)$ \\
\hline ACE inhibitors/ARBs & $863(35.0)$ \\
\hline Calcium-channel blockers & $972(39.4)$ \\
\hline Digoxin & $157(6.4)$ \\
\hline Diuretics & $513(20.8)$ \\
\hline
\end{tabular}

Continued
Table 3 Continued

Characteristics

KiCS-AF, $n=2464$, no. $(\%)$

Currently using antiarrhythmic drugs

$\begin{array}{lr}\text { Overall } & 526(21.3) \\ \text { Cibenzoline } & 66(2.7) \\ \text { Disopyramide } & 29(1.2) \\ \text { Pilsicainide } & 190(7.7) \\ \text { Flecainide } & 63(2.6) \\ \text { Amiodarone } & 29(1.2) \\ \text { Bepridil } & 135(5.5)\end{array}$

Oral anticoagulants

\begin{tabular}{|c|c|}
\hline Overall & $2021(82.0)$ \\
\hline Warfarin & $384(15.6)$ \\
\hline \multicolumn{2}{|l|}{ Direct oral anticoagulants } \\
\hline Overall & $1637(66.4)$ \\
\hline Dabigatran & $265(10.8)$ \\
\hline Rivaroxaban & $628(25.5)$ \\
\hline Apixaban & $630(25.6)$ \\
\hline Edoxaban & $114(4.6)$ \\
\hline Concomitant antiplatelet therapy & $316(12.8)$ \\
\hline $\begin{array}{l}\text { Oral anticoagulation in those with } \\
\mathrm{CHA}_{2} \mathrm{DS}_{2} \text {-VASc score }>2\end{array}$ & $1439(88.6)$ \\
\hline \multicolumn{2}{|l|}{ Prior interventional therapy for AF } \\
\hline Catheter ablation of $\mathrm{AF}$ & $160(6.5)$ \\
\hline $\mathrm{AV}$ node/His bundle ablation & $16(0.6)$ \\
\hline Surgical maze & $7(0.3)$ \\
\hline Rhythm control strategy & $1319(53.5)$ \\
\hline Rate control strategy & $1126(45.7)$ \\
\hline
\end{tabular}

$A C E$, angiotensin-converting enzyme; $A R B$, angiotensin receptor blocker;AV node, atrioventricular node; BMI, body mass index; BNP, brain natriuretic peptide;bpm, beats per minute; CABG, coronary artery bypass grafting; CKD, chronic kidney disease;eGFR, estimated glemerular filtration rate; HD, haemodialysis;KiCS-AF, the Keio interhospital Cardiovascular Studies-atrial fibrillation; LA, left atrium; LVEF, left ventricular ejection fraction; $\mathrm{PCI}$, percutaneous coronary intervention; TIA, transient ischaemic attack.

actual impact of screen-detected AF on both clinical and PROs will inform AF practice and further optimise care.

In addition, regarding the management strategy of $\mathrm{AF}$, NOACs were more commonly used in Japan compared with previous reports from Western countries. ${ }^{20}$ This finding may be attributable to the following: (1) prescriptions are covered by the National Health Insurance system in Japan ${ }^{12}$; (2) physicians may readily prescribe medications, as there are no pre-specified restrictions and (3) a growing body of data suggests that NOACs may be preferred for East Asian populations. ${ }^{21}$ It has been widely recognised that Asian patients treated with OAC therapy are known to have a higher risk of bleeding complications, and a much lower proportion of time-in-therapeutic 
Table 4 Characteristics of AF registries that obtained quality-of-life measures

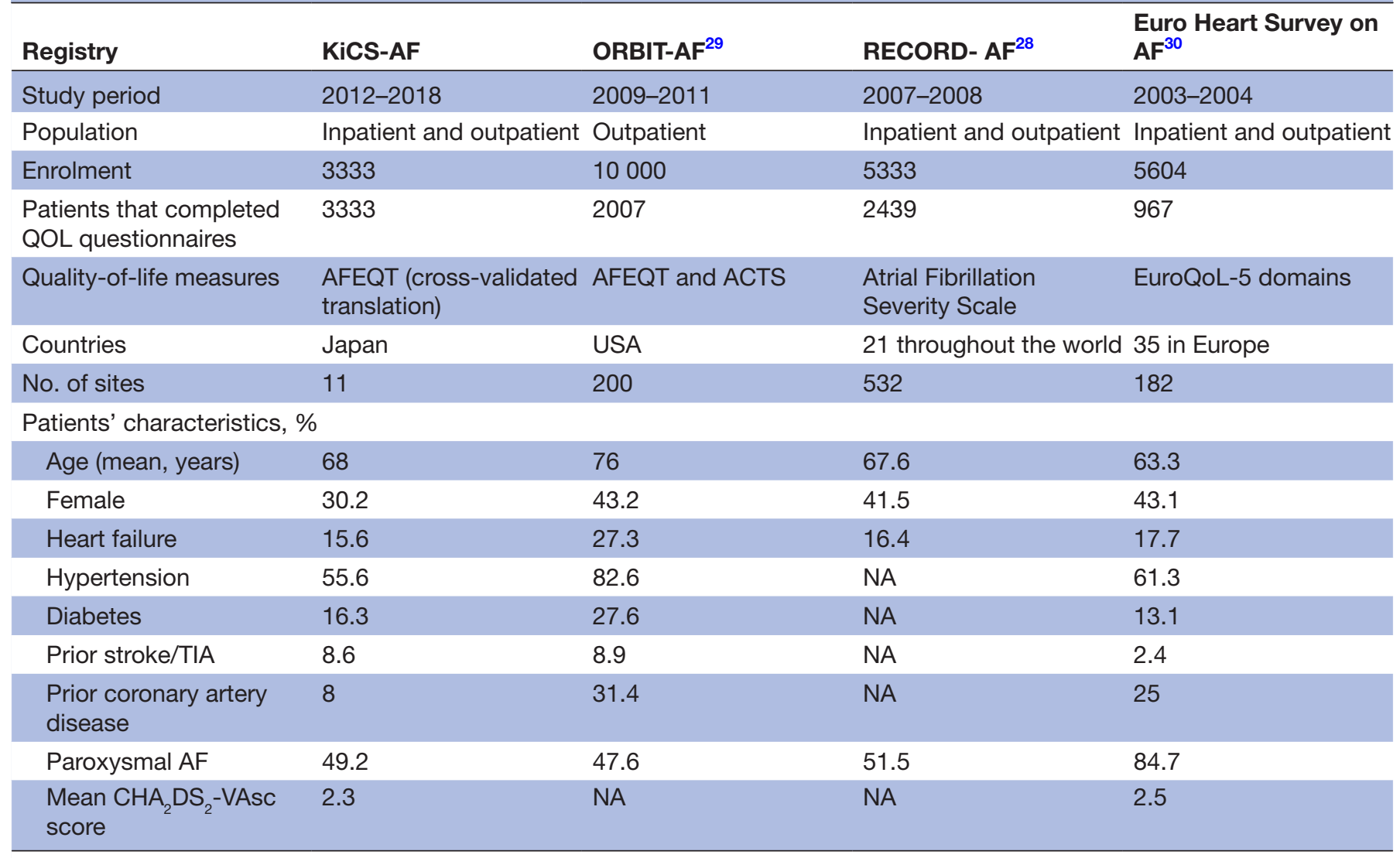

ACTS, anticoagulation-related quality-of-life assessment transient ischaemic attack; AF, atrial fibrillation; AFEQT, Atrial Fibrillation Effect on Quality-of-Life;KiCS-AF, the Keio interhospital Cardiovascular Studies-atrial fibrillation; ORBIT-AF, Outcomes Registry for Better Informed Treatment of Atrial Fibrillation;QOL, quality-of-life; RECORD-AF, Registry on Cardiac Rhythm Disorders Assessing the Control of Atrial Fibrillation; TIA, transient ischaemic attack.

range $(44 \%-68 \%)$ with warfarin compared with Western data was reported. ${ }^{22} 23$

\section{Symptoms and QOL}

The distribution of AF related symptoms is reported in figure 2. The most common symptoms that patients complained of at registration were palpitations $(41.3 \%)$

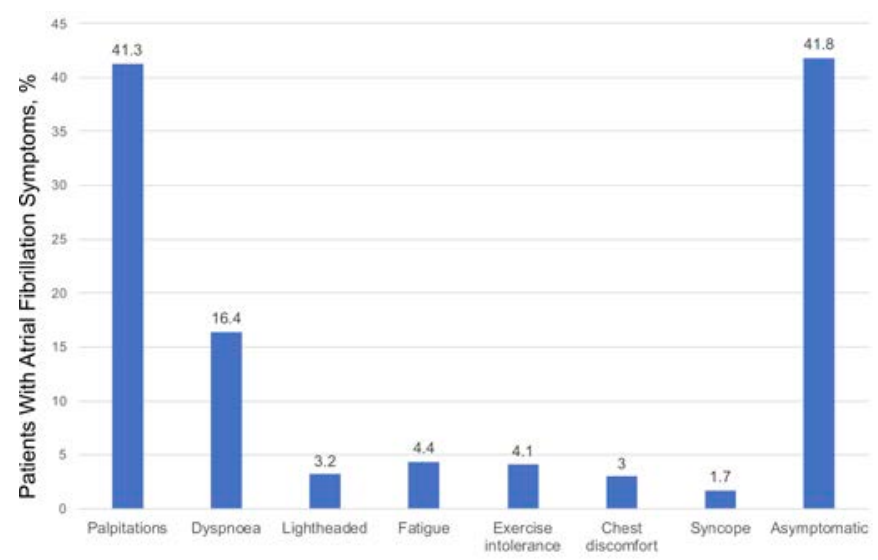

Figure 2 Distribution of symptoms for atrial fibrillation at the time of registration. Each bar shows atrial fibrillation symptom frequencies. followed by dyspnoea $(16.4 \%)$, while $41.8 \%$ of patients were asymptomatic at the time of registration. The median AFEQT-overall summary score was 79.1 (IQR, 66.6-89.1). A breakdown of each of the four domains is described in figure 3. Importantly, these results suggest that roughly $50 \%$ of the patients in the KiCS-AF had an impaired QOL at baseline. ${ }^{24}$ In addition, regardless of differences in patients' backgrounds, our findings regarding baseline AFEQT-overall summary score are in line with that of previous reports from the ORBIT-AF registry. ${ }^{4}$ Further investigations aimed at providing care and its association with patient-reported QOL may aid the development of a roadmap for global AF-related quality improvement and educational initiatives.

\section{STRENGTHS AND LIMITATIONS}

The KiCS-AF provides detailed data on the demographics, risk profiles, patterns of care and subsequent outcomes (including PROs) of contemporary Japanese patients with $\mathrm{AF}$ and, thus far, has revealed several important differences compared with patients with AF in Western countries. It is important to determine the clinical characteristics and profiles of patients with $\mathrm{AF}$ in different 


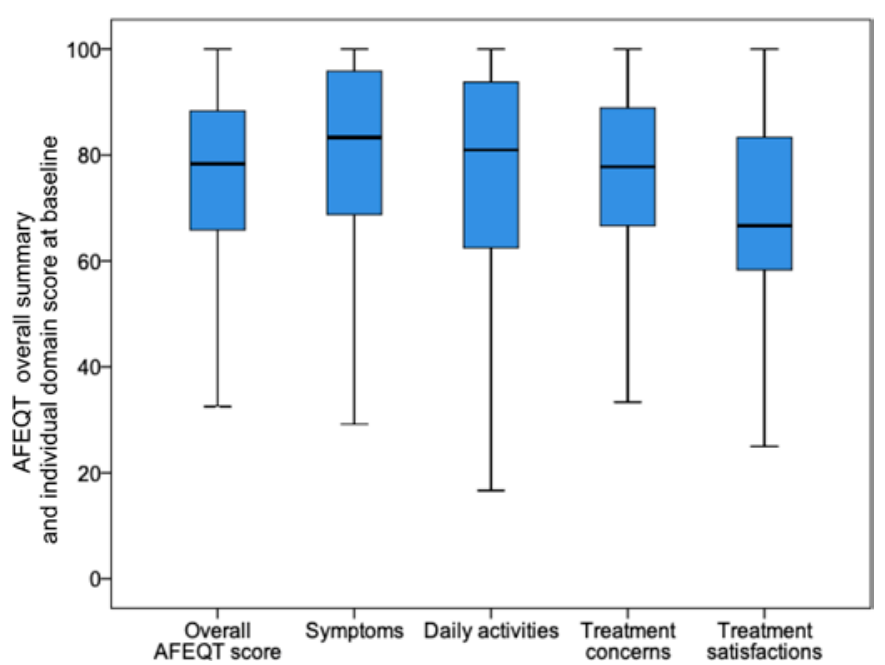

Figure 3 AFEQT survey results at the time of registration. The dark line in the middle of the boxes is the median of each score. The bottom of the box indicates the 25th percentile, and the top of the box represents the 75th percentile.

Approximately $95 \%$ of the data are expected to lie between the inner fences. AFEQT, Atrial Fibrillation Effect on Qualityof-Life.

regions. Such data are necessary for benchmarking, and enables comparisons across different countries, populations and healthcare systems.

Prior registries in Japan have examined the management of patients with AF mainly focused on their clinical outcomes. ${ }^{25-27}$ Although these and other registries have advanced our knowledge of AF, they have been limited by relatively old studies from a non-NOAC predominant era (ie, dabigatran was approved by Pharmaceuticals and
Medical Devices Agency in Japan in 2011) and lack of assessments on PROs (table 5). The KiCS-AF enrolled 3333 patients with a planned follow-up period of minimum 2 years (up to 5 years) to examine contemporary management of patients with $\mathrm{AF}$ in relation to patient-reported QOL outcomes. This long-term follow-up had several benefits, including a greater detection of infrequent clinical outcomes (eg, death and stroke) and the opportunity to observe trends in treatment strategies and their impacts on patient-reported QOL and other important outcomes. Furthermore, to the best of our knowledge, the KiCS-AF registry is the first study of $\mathrm{AF}$ to focus on PROs in the Asia Pacific region. AF is associated with significantly impaired QOL. ${ }^{3}$ Accordingly, many treatment decisions in AF are intended towards preserving and/or improving patients' reported QOL. Although, prior studies of QOL have been informative but limited by several aspects, such as restricted follow-up, ${ }^{28}$ relatively small sample size ${ }^{29}$ and the lack of disease-specific qualify-of-life questionnaires (table 4$).^{30}$

For a thorough understanding of our results, several limitations should be acknowledged. First, nonrandomised observational research involves inherent limitations, although it is the best way to describe the current treatment patterns and outcomes of care. There is likely to be unmeasured confounding, such as depression, frailty or economic status that may impact on the PROs. Second, not all AF patients in Japan participated in the KiCS-AF registry, and the sampling bias and generalisability of the study results to Japan is a potential concern, although we were very inclusive of those presenting to the participating centres with new-onset AF. As patients

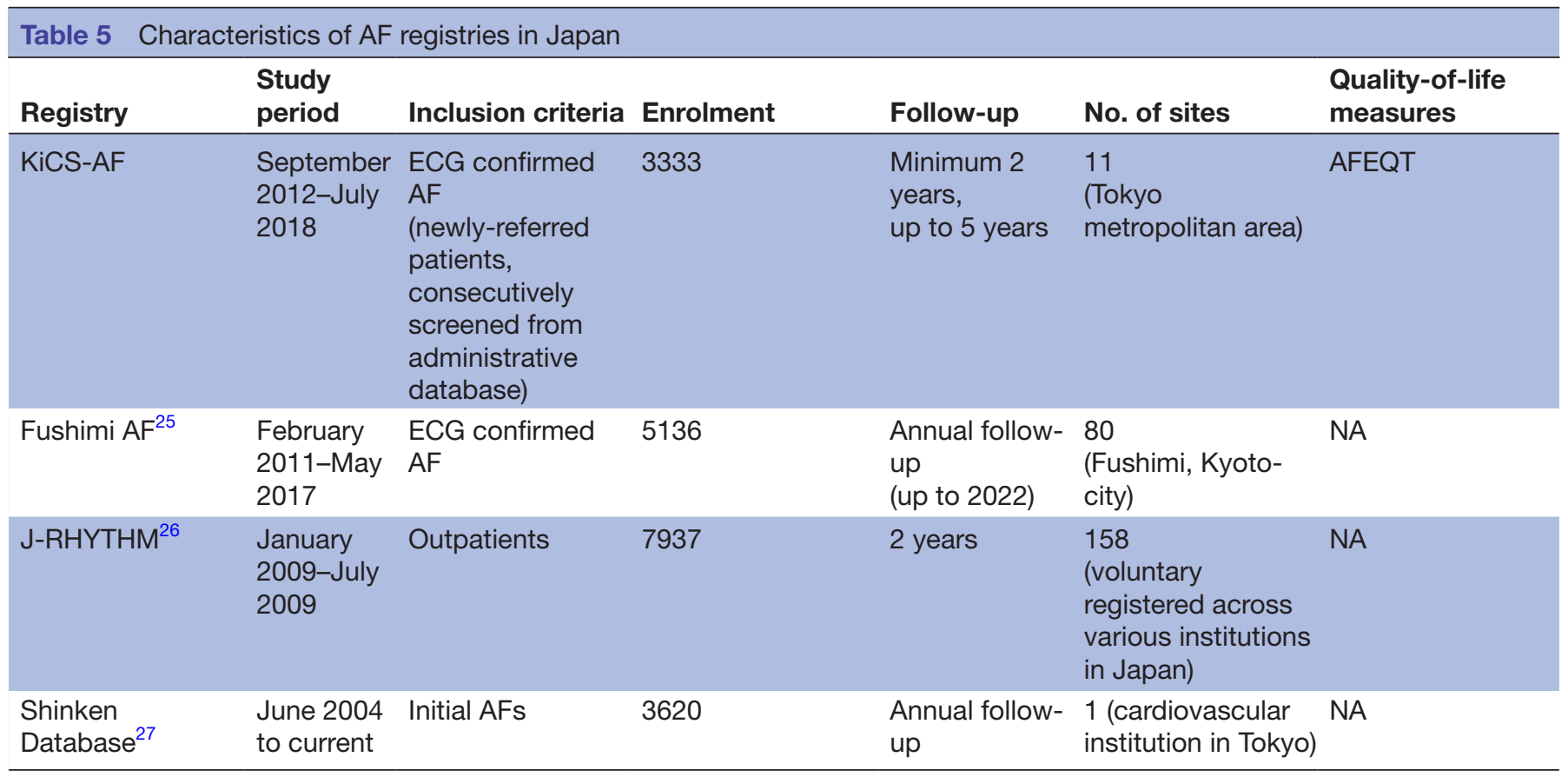

AF, atrial fibrillation; AFEQT, Atrial Fibrillation Effect on Quality-of-Life;KiCS-AF, Keio interhospital Cardiovascular Studies-atrial fibrillation; NA, not applicable. 
were primarily referred to large tertiary care referral centres in the Tokyo metropolitan area, selection bias may result in patients with $\mathrm{AF}$ at the outpatient clinical level in more rural areas or patient who do not require intensive cardiovascular care not being captured. Despite this potential limitation, we believe that this is one of the most representative Japanese databases of AF patients, and our results comprise the most complete assessment of current practice patterns and QOL outcomes in Japan. Third, echocardiograms were not performed as part of the study protocol for clinical reasons, and thus standardised echocardiograph protocols have not been used across all participating hospitals.

In conclusion, the KiCS-AF allowed for extensive investigation of AF-related QOL in a non-selected population over a long-term follow-up by using a rigorously validated QOL assessment tool. Our future analyses will be aimed at determining if QOL differs according to patients' backgrounds, cardiac biomarkers, echocardiography data or treatment strategies. Additional analyses will assess whether these differences in AF-related QOL are associated with differences in care providers' decisions towards implementation of rhythm control or anticoagulation strategies in routine clinical practice. In addition, QOL assessments with respect to anticoagulation therapy may aid in improvement of patient satisfaction, adherence and better physician-patient relationship. Finally, these data could also serve as a reference for indirect comparisons of QOL in experimental studies or comparative effectiveness research. ${ }^{29}$

\section{COLLABORATION}

The data and materials used to conduct this research are available to researchers, on request, for scientific projects aimed at identifying a novel clinical finding that may further improve patient outcome. Attempts to co-validate country-specific observations, risk stratification schemes, and outcomes are also welcome. The procedure does need to follow the Act on the Protection of Personal Information Law (as of May 2017) and the Ethical Guidelines for Medical and Health Research Involving Human Subjects (as of March 2015) in Japan.

\footnotetext{
Author affiliations

${ }^{1}$ Department of Cardiology, Keio University School of Medicine, Tokyo, Japan

${ }^{2}$ Cardiovascular Research, Department of Biomedical and Health Informatics, Saint

Luke's Mid America Heart Institute, Kansas City, Missouri, USA

${ }^{3}$ Stanford Center for Clinical Research, Stanford University School of Medicine,

Stanford, California, USA

${ }^{4}$ Duke Clinical Research Institute, Duke University Medical Center, Durham, North Carolina, USA

${ }^{5}$ Division of Cardiology, Vancouver General Hospital, Vancouver, British Columbia, Canada

${ }^{6}$ Department of Cardiology, National Hospital Organisation Tokyo Medical Center, Meguro-ku, Tokyo, Japan

${ }^{7}$ Department of Cardiology, National Hospital Organization Saitama Hospital, Wako, Japan

${ }^{8}$ Department of Cardiology, Hino Shiritsu Byoin, Hino, Tokyo, Japan

${ }^{9}$ Department of Cardiology, Tokai University Tokyo Hospital, Shibuya-ku, Tokyo, Japan
}

${ }^{10}$ Department of Cardiology, Tachikawa Hospital, Tachikawa, Tokyo, Japan

Twitter Nobuhiro Ikemura @Nobu0129 and Taku Inohara @taku_inohara

Acknowledgements We are grateful to all study coordinators, investigators and patients who participated in the KiCS-AF registry.

Collaborators Site investigators: Munehisa Sakamoto, Jun Fuse, Kojiro Tanimoto, Yoko Tanimoto, Yukinori lkegami, Kohei Inagawa (National Hospital Organization Tokyo Medical Center). Jyunji Suzuki, Tomohiro Matsuhashi, Hiroshi Shiga (Hino Municipal Hospital). Yoshiyasu Aizawa, Nobuhiro Nishiyama, Takahiko Nishiyama, Yoshinori Katsumata, Shin Kashimura, Akira Kunitomi, Kazuaki Nakajima, Taishi Fujisawa (Keio University School of Medicine). Takaharu Katayama, Keisuke Matsumura, Tomohiko Ono, Hanako Tokuda, Ryutaro Yamaguchi, Hiroaki Tanaka (National Hospital Organization Saitama National Hospital). Takashi Yagi, Kenichiro Shimoji, Koji Ueno, Satoshi Mogi (Saiseikai Utsunomiya Hospital). Shiro Ishikawa, Hideaki Kanki, Takashi Akima, Masahito Munakata, Kazutaka Miyamoto (Saitama City Hospital). Kazunori Moritani, Masaru Shibata, Toshimi Kageyama (Tachikawa Hospital). Akiyasu Baba, Yoshinori Mano, Hiroaki Sukegawa (Tokyo Dental College Ichikawa General Hospital). Takahiro Koura, Daisuke Shinmura, Kotaro Fukumoto, Hiroyuki Yamakawa (Yokohama Municipal Citizen's Hospital). Kazuhiro Oyamada, Kotaro Naitou, Keijiro Chiba (Keiyu Hospital). Clinical co-ordinators: Aki Kato, Ikumi Koishi, Miho Matsuoka, Takako Nozaki, Hiroaki Nagayama, Chieko Tamura, Reiko Tamura, Junko Susa, Miho Umemura and Itsuka Saito.

Contributors NI, SK and TK had full access to all data in the study and take responsibility for the integrity of the data and the accuracy of the data analysis. IU, $\mathrm{KT}, \mathrm{MS}$, IN, MA and HM contributed substantially to data collection. NI, JAS, TK, KM, JPP, TI, KF, ST and SK contributed substantially to the study design, data analysis, data interpretation and the writing of the manuscript.

Funding This study was funded by a Grant-in-Aid for Scientific Research from the Japan Society for the Promotion of Science (grant no. 16H05215, 16KK0186) and by an unrestricted research grant from Bayer Yakuhin Ltd.

Map disclaimer The depiction of boundaries on this map does not imply the expression of any opinion whatsoever on the part of BMJ (or any member of its group) concerning the legal status of any country, territory, jurisdiction or area or of its authorities. This map is provided without any warranty of any kind, either express or implied.

Competing interests SK received an unrestricted research grant for the Department of Cardiology at Keio University School of Medicine from Bayer Pharmaceutical and Daiichi Sankyo; reported receiving grants from Bayer Yakuhin Ltd and Daiichi Sankyo; and reported receiving personal fees from Bristol-Myers Squibb. TK received grants from Bayer Yakuhin Ltd. JS received personal fees from Novartis, AstraZeneca, Janssen, Bayer, Boehringer Ingelheim, Regeneron, Corvia and United Healthcare; reported receiving grants from Bayer and Abbott Vascular; and reported owning equity in Health Outcomes Sciences. ST received grants and personal fees from Bayer and reported receiving personal fees from Daiichi Sankyo and Bristol-Myers Squibb.

Patient consent for publication Not required.

Ethics approval The ethical review board at each hospital reviewed and approved the study protocol; the list of ethical review board which approve the study was available in the online supplementary material. The institutional review board at Keio University School of Medicine reviewed and approved the study protocol (ID: 20120029).

Provenance and peer review Not commissioned; externally peer reviewed.

Data availability statement Data are available upon reasonable request.

Open access This is an open access article distributed in accordance with the Creative Commons Attribution Non Commercial (CC BY-NC 4.0) license, which permits others to distribute, remix, adapt, build upon this work non-commercially, and license their derivative works on different terms, provided the original work is properly cited, appropriate credit is given, any changes made indicated, and the use is non-commercial. See: http://creativecommons.org/licenses/by-nc/4.0/.

ORCID iD

Nobuhiro Ikemura http://orcid.org/0000-0002-6347-4460 


\section{REFERENCES}

1 Chugh SS, Havmoeller R, Narayanan K, et al. Worldwide epidemiology of atrial fibrillation: a global burden of disease 2010 study. Circulation 2014;129:837-47.

2 Wang TJ, Larson MG, Levy D, et al. Temporal relations of atrial fibrillation and congestive heart failure and their joint influence on mortality: the Framingham heart study. Circulation 2003;107:2920-5.

3 Freeman JV, Simon DN, Go AS, et al. Association between atrial fibrillation symptoms, quality of life, and patient outcomes: results from the outcomes Registry for better informed treatment of atrial fibrillation (ORBIT-AF). Circ Cardiovasc Qual Outcomes 2015;8:393-402

4 Randolph TC, Simon DN, Thomas L, et al. Patient factors associated with quality of life in atrial fibrillation. Am Heart J 2016;182:135-43.

5 Stewart S, Murphy N, Walker A, et al. Cost of an emerging epidemic: an economic analysis of atrial fibrillation in the UK. Heart 2004:90:286-92.

$6 \mathrm{Kim} \mathrm{MH}$, Johnston SS, Chu B-C, et al. Estimation of total incremental health care costs in patients with atrial fibrillation in the United States. Circ Cardiovasc Qual Outcomes 2011;4:313-20.

7 Go AS, Hylek EM, Phillips KA, et al. Prevalence of diagnosed atrial fibrillation in adults: national implications for rhythm management and stroke prevention: the anticoagulation and risk factors in atrial fibrillation (atria) study. JAMA 2001;285:2370-5.

8 Inoue $\mathrm{H}$, Fujiki $\mathrm{A}$, Origasa $\mathrm{H}$, et al. Prevalence of atrial fibrillation in the general population of Japan: an analysis based on periodic health examination. Int J Cardiol 2009;137:102-7.

9 Chiang C-E, Zhang S, Tse HF, et al. Atrial fibrillation management in Asia: from the Asian expert forum on atrial fibrillation. Int $J$ Cardiol 2013;164:21-32.

10 January CT, Wann LS, Alpert JS, et al. 2014 AHA/ACC/HRS guideline for the management of patients with atrial fibrillation: a report of the American College of Cardiology/American heart association Task force on practice guidelines and the heart rhythm Society. Circulation 2014;130:e199-267.

11 Lip GYH, Brechin CM, Lane DA. The global burden of atrial fibrillation and stroke: a systematic review of the epidemiology of atrial fibrillation in regions outside North America and Europe. Chest 2012;142:1489-98.

12 Ikegami N, Yoo B-K, Hashimoto $\mathrm{H}$, et al. Japanese universal health coverage: evolution, achievements, and challenges. Lancet 2011;378:1106-15

13 Gage BF, Waterman AD, Shannon W, et al. Validation of clinical classification schemes for predicting stroke: results from the National Registry of atrial fibrillation. JAMA 2001;285:2864-70.

14 Lip GYH, Nieuwlaat R, Pisters R, et al. Refining clinical risk stratification for predicting stroke and thromboembolism in atrial fibrillation using a novel risk factor-based approach: the Euro heart survey on atrial fibrillation. Chest 2010;137:263-72.

15 Spertus J, Dorian P, Bubien R, et al. Development and validation of the atrial fibrillation effect on quality-of-life (AFEQT) questionnaire in patients with atrial fibrillation. Circ Arrhythm Electrophysiol 2011;4:15-25.
16 Kaatz S, Ahmad D, Spyropoulos AC, et al. Definition of clinically relevant non-major bleeding in studies of anticoagulants in atrial fibrillation and venous thromboembolic disease in non-surgical patients: communication from the SSC of the ISTH. J Thromb Haemost 2015;13:2119-26.

17 Hori M, Matsumoto M, Tanahashi N, et al. Rivaroxaban vs. warfarin in Japanese patients with atrial fibrillation - the J-ROCKET AF study -. Circ J 2012;76:2104-11.

18 Ogawa S, Yamashita T, Yamazaki T, et al. Optimal treatment strategy for patients with paroxysmal atrial fibrillation: J-RHYTHM study. Circ $J$ 2009;73:242-8.

19 Golwala H, Jackson LR, Simon DN, et al. Racial/Ethnic differences in atrial fibrillation symptoms, treatment patterns, and outcomes: insights from outcomes Registry for better informed treatment for atrial fibrillation registry. Am Heart J 2016;174:29-36.

20 Steinberg BA, Gao H, Shrader P, et al. International trends in clinical characteristics and oral anticoagulation treatment for patients with atrial fibrillation: results from the GARFIELD-AF, ORBIT-AF I, and ORBIT-AF II registries. Am Heart J 2017;194:132-40.

21 Chiang C-E, Wang K-L, Lin S-J. Asian strategy for stroke prevention in atrial fibrillation. Europace 2015;17:ii31-9.

22 Shen AY-J, Yao JF, Brar SS, et al. Racial/ethnic differences in the risk of intracranial hemorrhage among patients with atrial fibrillation. $J$ Am Coll Cardiol 2007;50:309-15.

23 Wallentin L, Yusuf S, Ezekowitz MD, et al. Efficacy and safety of dabigatran compared with warfarin at different levels of international normalised ratio control for stroke prevention in atrial fibrillation: an analysis of the RE-LY trial. The Lancet 2010;376:975-83.

24 Wynn GJ, Todd DM, Webber M, et al. The European heart rhythm association symptom classification for atrial fibrillation: validation and improvement through a simple modification. Europace 2014;16:965-72.

25 Chishaki $\mathrm{A}$, Chishaki $\mathrm{H}$. To know the exact prevalence and prognosis of atrial fibrillation from a clinical survey--comments on the "The Fushimi AF Registry". J Cardiol 2013;61:304-6.

26 Atarashi $\mathrm{H}$, Inoue $\mathrm{H}$, Okumura $\mathrm{K}$, et al. Investigation of optimal anticoagulation strategy for stroke prevention in Japanese patients with atrial fibrillation--the J-RHYTHM Registry study design. $J$ Cardiol 2011;57:95-9.

27 Suzuki S, Yamashita T, Ohtsuka T, et al. Prevalence and prognosis of patients with atrial fibrillation in Japan: a prospective cohort of Shinken database 2004. Circ J 2008;72:914-20.

$28 \mathrm{Ha}$ ACT, Breithardt G, Camm AJ, et al. Health-related quality of life in patients with atrial fibrillation treated with rhythm control versus rate control: insights from a prospective international registry (registry on cardiac rhythm disorders assessing the control of atrial fibrillation: RECORD-AF). Circ Cardiovasc Qual Outcomes 2014;7:896-904.

29 Piccini JP, Fraulo ES, Ansell JE, et al. Outcomes registry for better informed treatment of atrial fibrillation: rationale and design of ORBIT-AF. Am Heart J 2011;162:606-12.

30 Dudink EAMP, Erküner Ömer, Berg J, et al. The influence of progression of atrial fibrillation on quality of life: a report from the Euro heart survey. Europace 2018;20:929-34. 